\title{
EFEITOS DA VINHOTERAPIA EM PARÂMETROS SANGUÍNEOS. (COLESTEROL, GLICOSE E TRIGLICERÍDEOS)
}

\section{EFFECTS OF WINE IN SANGUINE PARAMETERS. (CHOLESTEROL, GLYCERIN AND TREEGLICERIDS)}

\author{
SILVA, J1; SALVINI, D. V.2; \\ 1. Prof. Espec. do Departamento de Ciências Biológicas da FAG.; \\ 2. Graduanda de Ciências Biológicas Bacharelado pela Faculdade Assis \\ Gurgacz; e-mail: josnei@ fag.edu.br REC:02/09 AC: 03/09 \\ Trabalho de Conclusão de Curso (Graduação em Ciências Biológicas) - Curso de Ciências Biológicas \\ Bacharelado, Faculdade Assis Gurgacz, Cascavel, 2009.
}

\begin{abstract}
RESUMO
É expressivo o número de evidências acerca das propriedades benéficas do vinho para a saúde humana. Estudos recentes comprovaram que certas substâncias presentes no vinho, os compostos fenólicos, possuem atividade antioxidante inibindo a oxidação do LDL - Colesterol. Além da ação antioxidante, mecanismos antiplaquetários, antiinflamatórios e vasodilatadores. Segundo estudos, o etanol eleva os níveis sangüíneos de HDL - Colesterol também faz parte das propriedades do vinho. Entretanto, por ser uma bebida alcoólica, é importante ressaltar que o vinho deve ser consumido com moderação, sendo que o consumo excessivo passa a ser fator de diversas doenças. O presente estudo analisou trinta pacientes com alimentação normal, ingerindo vinho tinto Cabernet Sauvignon, Isabel e Chardonnay durante a refeição principal, num período de trinta dias, acompanhado por exames sanguíneos: colesterol total, HDLColesterol, LDL- Colesterol, triglicerídeos e glicemia pré-vinho e pós-vinho. Com relação aos exames laboratoriais estes apresentaram um aumento significativo no HDL - Colesterol $(p<0,05)$ apenas com o grupo que teve a ingestão do vinho Isabel.
\end{abstract}

Palavras-chave: HDL - colesterol. Saúde. Vinho.

\begin{abstract}
It is expressive the number of evidences concerning the beneficial properties of the wine for the human health. Recent studies proved that certain present substances in the wine, the compositions phenolic, possess antioxidant activity inhibiting the oxidation of LDL - Cholesterol. Besides the antioxidant action, mechanisms anti-platelet, anti-inflammatory and vasodilator. According to studies, the ethanol elevates the levels sanguine of HDL - Cholesterol, they are also part of the properties of the wine. However, for being an alcoholic drink, it is important to point out that the wine should be consumed with moderation, and the excessive consumption becomes factor of several diseases. The present study analyzed thirty patients with normal feeding, ingesting red wine Cabernet Sauvignon, Isabel and Chardonnay during the main meal, in a period of thirty days, accompanied by sanguine exams: total cholesterol, HDL - Cholesterol, LDL - Cholesterol, treeglicerids and glycerin per-wine and powder-wine. With relationship to the exams laboratories these presented a significant increase in HDL - Cholesterol $(p<0,05)$ just with the group that had the ingestion of the wine Isabel.
\end{abstract}

Word-key: HDL - cholesterol. Health. Wine. 


\section{INTRODUÇÃO}

Inúmeras pesquisas comprovam que o vinho é a bebida mais favorável a saúde, isso se ingerido junto com as refeições, regularmente, com moderação e por quem não tem contra-indicação a ingesta de bebidas alcoólicas (SOUZA FILHO, 2002). Não é possível afirmar com certeza a origem do vinho, mas alguns registros históricos indicam que foi há cerca de 6000 anos, no Oriente, na região do Cáucaso, onde se localizam atualmente a Geórgia e a Armênia (JOHNSON, 2004).

Classicamente, o vinho é definido como uma bebida natural, biológica, resultante da fermentação alcoólica e parcial de uvas frescas, ligeiramente amadurecidas, ou de seus mostos, onde a graduação alcoólica natural nunca será inferior a nove graus (nove por cento em volume) exceto em vinhos verdes (BLASI, 2004).

As uvas que originam os melhores vinhos são da espécie Vitis vinifera, de origem européia, que possui inúmeras castas, entre as quais se destacam: Cabernet Sauvignon, Merlot, Pinot Noir, Syrah e Chardonnay. As demais espécies são de origem americana e, em geral, não são adequadas para a elaboração de vinhos, prestando-se mais como uvas de mesa e suco. Essas espécies também possuem muitas variedades, cujos melhores exemplos no Brasil são a Niágara e Isabel, que até a década de 80 eram as únicas castas utilizadas na elaboração dos vinhos brasileiros (LONA, 2001).

A capacidade antioxidante total dos vinhos tintos é muito superior a dos vinhos brancos, uma vez que nestes a concentração de polifenóis é de cerca de $250 \mathrm{mg} / \mathrm{L}$, ao passo que em vinhos tintos estes valores oscilam entre 1000 e $4000 \mathrm{mg} / \mathrm{L}$ (WATERHOUSE, 2002).

Neste sentido, a capacidade antioxidante e a concentração total de polifenóis de um cálice de vinho tinto $(150 \mathrm{~mL})$ equivalem a doze cálices de vinho branco, dois copos de chá, quatro maçãs e sete copos de suco de laranja (ISHIMOTO, et al., 2006). O vinho com uvas Cabernet sauvignon possui concentração de polifenóis totais oito vezes maior que a do vinho branco, explicação plausível para sua maior capacidade removedora de radicais peroxinitrito, potentes oxidantes em sistemas biológicos (FERRARI, 2001).

Certas substâncias presentes no vinho, os compostos fenólicos, possuem atividade antioxidante, inibindo a oxidação celular do LDL colesterol e HDL colesterol, além da ação antioxidante, mecanismos antiplaquetários, antiinflamatórios e vasodilatadores (MAGALHÃES ANDRADE 2006) adesão endotelial, supressão de crescimento de células do câncer que também foram atribuídos aos compostos fenólicos do vinho (DE LORIMIER, 2000).

Os flavonóides do vinho tinto exercem efeito maior contra a oxidação da LDL do que o suco de uva, ou vinho branco. Pesquisas com humanos mostraram que a concentração de polifenóis no plasma aumentou significativamente após a ingestão de vinho tinto. A concentração de catequina (+), o constituinte fenólico mais abundante do vinho tinto, aumentou de $2 \mathrm{nmol} / \mathrm{L}$ para $91 \pm 14 \mathrm{nmol} / \mathrm{L}$ em nove voluntários saudáveis após o consumo de $120 \mathrm{~mL}$ de vinho tinto (LINDBERG, 2008).

Entretanto, o consumo diário de vinho deve ser controlado para não causar doenças como úlcera gastrointestinal, alcoolismo e a cardiopatia alcoólica (OLIVEIRA MAMEDE, 2004). 
O consumo de até $49 \mathrm{~g} /$ dia de álcool em consumidores de vinho foi associado com um aumento de HDL colesterol e apolipoiproteína Al, e não de triglicérides (Parret et al, 2002). Além dos efeitos sobre os componentes gordurosos do plasma, o vinho, via álcool, também parece poder atuar na prevenção da $\mathrm{DCl}$, por meio de interferência nos mecanismos de coagulação sanguínea (BLASI, 2004).

Não há um consenso mundial sobre a definição de "consumo moderado". Porém consumo moderado de álcool pode ser definido como sendo a ingestão de 1 a 2 doses de drinks por dia, sendo que 1 dose corresponde a 150mL de vinho. Este critério é o mesmo utilizado pela Organização Mundial de Saúde, que recomenda o consumo de até 30 gramas de álcool por dia, desde que não haja contra-indicação no uso de bebidas alcoólicas. Assim, na ausência de contra-indicação, o consumo diário de 200 a 300mL de vinho tinto (com 12\% de graduação alcoólica) pode ser considerado um fator de proteção à saúde cardiovascular. (ISHIMOTO, et.al, 2006).

Em doses altas, o álcool atua como forte depressor do miocárdio e tem efeito tóxico direto sobre o mesmo ("doença cardíaca alcoólica"). A ação do álcool como vasodilatador coronariano é controversa, mas a caso de crises de angina em que o álcool pode gerar crise de alívio da dor sem modificar as alterações isquêmicas, surgindo um efeito depressor no SNC (BLASI, 2004).

O presente trabalho teve como objetivo comparar os efeitos do vinho com uvas Cabernet Sauvignon, Chardonnay e Isabel, sobre os parâmetros sanguíneos: glicemia, colesterol total, HDL - Colesterol, LDL - Colesterol e triglicerídeos.

\section{MATERIAL E MÉTODOS}

Trinta indivíduos assinaram o termo de consentimento esclarecido (APÊNDICE A) e uma anamnese inicial (APÊNDICE B) aprovado pelo Comitê de Ética em Pesquisa (parecer 10/2009) (APÊNDICE C) da Faculdade Assis Gurgacz. Completaram o estudo vinte e nove indivíduos que foram divididos em três grupos: vinho tinto nobre (uvas Cabernet Sauvignon), vinho branco nobre (uvas Chardonnay) e vinho tinto colonial (uvas Isabel). Foram analisados indivíduos com idade entre vinte a cinqüenta e quatro anos, pessoas saudáveis com alimentação variável (sem dieta controlada).

Solicitou-se a todos que se abstivessem de bebidas alcoólicas (outras que não o vinho fornecido durante a pesquisa), de qualquer substância ou bebida que possuísse como ingredientes: cafeína, polifenóis (por exemplo: frutas com casca escura como maçã, uva, ameixa; chás, como chá verde ou mate), cebola, alho e chocolate, por no mínimo 72 horas antes dos exames, estando tais alimentos liberados durante os outros momentos da pesquisa. Os indivíduos foram orientados a manter a mesma rotina em relação à dieta e à atividade física em todos os momentos, tanto antes e durante o consumo de vinho.

Foram realizados exames sanguíneos em dias previamente agendados, sendo um inicial e um final após trinta dias da ingesta do vinho. O plasma dos indivíduos foi colhido em tubo seco após jejum de 12 horas por venopunção para a realização das análises de LDL - colesterol, HDL - colesterol, colesterol total, triglicerídeos, glicemia e Gama-Glutil-Transferase. As análises foram realizadas em laboratório particular 
(Unilabor, Cascavel-PR).

Os vinhos utilizados foram o Varietais, com uvas Cabernet Sauvignon, uvas

Chardonnay e o vinho colonial com uvas Isabel. Os pacientes foram orientados a ingerir $100 \mathrm{~mL}$ de vinho durante uma refeição principal. Oarmazenamento foi realizado pelos próprios voluntários, sendo fornecido a cada um o total de 8 garrafas de vinho de 375 $\mathrm{ml}$. Os indivíduos eram questionados acerca da ingestão correta do vinho, durante os trinta dias da pesquisa. Apenas uma paciente não completou o estudo, pôs apresentou sintomas de gastrite, sendo orientada a encerrar a ingesta de vinho.

Os dados foram expressos como média + desvio padrão. As variáveis foram comparadas, entre os grupos estudados, utilizando o programa MINITAB 15 para todas as análises estatísticas. Para efeito de gráficos, os dados foram expressos como média. $O$ valor de $p<0,05$ foi considerado estatisticamente significativo.

\section{RESULTADOS}

Verificou-se que os níveis lipídicos entre os grupos pré-vinho (TABELA 1) e pósvinho (TABELA2), apresentaram alterações lipídicas expressivas. Ao analisar as taxas lipídicas pós-vinho, demonstraram que o Colesterol Total do grupo que consumiu vinho de uvas Cabernet Sauvignon aumentou em 3\%, ao grupo de vinho com uva Isabel aumentou 5\%, enquanto que com uvas Chardonnay permaneceu igual, em comparação a análise pré-vinho (GRÁFICO 1).

TABELA 1: DADOS LABORATORIAIS, PERFIL LIPÍDICO, GLICEMIA E TRANSFERASE PRÉ-VINHO

\begin{tabular}{|c|c|c|c|c|c|c|}
\hline Grupo & $\begin{array}{c}\mathrm{CT} \\
(\mathrm{mg} / \mathrm{dl})\end{array}$ & $\begin{array}{c}\mathrm{TG} \\
(\mathrm{mg} / \mathrm{dl})\end{array}$ & $\begin{array}{c}\mathrm{HDL} \\
(\mathrm{mg} / \mathrm{dl})\end{array}$ & $\begin{array}{c}\mathrm{LDL} \\
(\mathrm{mg} / \mathrm{dl})\end{array}$ & $\begin{array}{l}\text { Glicose } \\
\text { (mg/dl) }\end{array}$ & $\begin{array}{c}\text { Gama GT } \\
(\mathrm{mg} / \mathrm{dl})\end{array}$ \\
\hline Isabel & 201 & 319 & 32 & 105 & 88 & 77 \\
\hline Isabel & 189 & 122 & 57 & 108 & 106 & 11 \\
\hline Isabel & 171 & 56 & 49 & 111 & 78 & 27 \\
\hline Isabel & 187 & 138 & 71 & 88 & 87 & 27 \\
\hline Isabel & 164 & 50 & 59 & 94 & 87 & 12 \\
\hline Isabel & 175 & 181 & 49 & 90 & 81 & 18 \\
\hline Isabel & 146 & 59 & 37 & 97 & 88 & 13 \\
\hline Isabel & 181 & 108 & 51 & 108 & 70 & 13 \\
\hline Isabel & 182 & 92 & 59 & 105 & 74 & 9 \\
\hline Isabel & 166 & 121 & 42 & 100 & 97 & 28 \\
\hline Cabernet & 139 & 59 & 42 & 85 & 82 & 10 \\
\hline Cabernet & 164 & 85 & 55 & 91 & 86 & 19 \\
\hline Cabernet & 181 & 85 & 45 & 119 & 103 & 50 \\
\hline Cabernet & 189 & 93 & 52 & 119 & 75 & 10 \\
\hline Cabernet & 176 & 146 & 42 & 104 & 88 & 14 \\
\hline Cabernet & 124 & 166 & 32 & 59 & 73 & 24 \\
\hline Cabernet & 171 & 106 & 68 & 82 & 90 & 12 \\
\hline Cabernet & 162 & 159 & 39 & 91 & 72 & 15 \\
\hline Cabernet & 216 & 99 & 67 & 130 & 85 & 10 \\
\hline Carbenet & 189 & 91 & 63 & 107 & 84 & 11 \\
\hline Chardonnay & 187 & 95 & 61 & 107 & 89 & 10 \\
\hline Chardonnay & 150 & 156 & 38 & 81 & 88 & 11 \\
\hline Chardonnay & 166 & 42 & 69 & 88 & 90 & 9 \\
\hline Chardonnay & 143 & 69 & 56 & 73 & 87 & 10 \\
\hline Chardonnay & 177 & 69 & 76 & 87 & 85 & 10 \\
\hline Chardonnay & 118 & 58 & 43 & 63 & 85 & 12 \\
\hline Chardonnay & 125 & 95 & 42 & 64 & 95 & 17 \\
\hline Chardonnay & 134 & 83 & 41 & 77 & 84 & 10 \\
\hline Chardonnay & 147 & 95 & 60 & 68 & 95 & 18 \\
\hline
\end{tabular}

$\overline{\mathrm{CT}}=$ colesterol total; $\mathrm{TG}=$ triglicerídeos; $\mathrm{HDL}=\mathrm{HDL}$-colesterol; $\mathrm{LDL}=\mathrm{LDL}$-colesterol; GAMA GT= Gama -

Glutil-Transferase 
TABELA 2: DADOS LABORAORIAIS, PERFIL LIPÍDICO, GLICEMIA E TRANSFERASE PÓS-VINHO

\begin{tabular}{|c|c|c|c|c|c|c|}
\hline Grupo & $\begin{array}{c}\text { CT } \\
(\mathrm{mg} / \mathrm{dl})\end{array}$ & $\begin{array}{c}\mathrm{TG} \\
(\mathrm{mg} / \mathrm{dl})\end{array}$ & $\begin{array}{c}\mathrm{HDL} \\
(\mathrm{mg} / \mathrm{dl})\end{array}$ & $\begin{array}{c}\text { LDL } \\
(\mathrm{mg} / \mathrm{dl})\end{array}$ & $\begin{array}{c}\text { Glicose } \\
\text { (mg/dl) }\end{array}$ & $\begin{array}{c}\text { Gama } \\
\text { GT } \\
(\mathrm{mg} / \mathrm{dl})\end{array}$ \\
\hline Isabel & 205 & 173 & 48 & 122 & 85 & 54 \\
\hline Isabel & 187 & 80 & 55 & 117 & 81 & 17 \\
\hline Isabel & 218 & 67 & 61 & 144 & 90 & 32 \\
\hline Isabel & 193 & 126 & 86 & 82 & 87 & 18 \\
\hline Isabel & 158 & 49 & 70 & 79 & 86 & 13 \\
\hline Isabel & 176 & 214 & 53 & 80 & 91 & 14 \\
\hline Isabel & 163 & 137 & 46 & 90 & 86 & 17 \\
\hline Isabel & 218 & 99 & 68 & 130 & 80 & 13 \\
\hline Isabel & 201 & 78 & 76 & 110 & 76 & 12 \\
\hline Isabel & 166 & 122 & 58 & 84 & 104 & 31 \\
\hline Cabernet & 136 & 44 & 49 & 78 & 94 & 15 \\
\hline Cabernet & 198 & 138 & 69 & 102 & 89 & 27 \\
\hline Cabernet & 210 & 194 & 55 & 116 & 104 & 89 \\
\hline Cabernet & 170 & 151 & 66 & 74 & 80 & 11 \\
\hline Cabernet & 196 & 138 & 62 & 107 & 83 & 18 \\
\hline Cabernet & 137 & 88 & 40 & 79 & 77 & 34 \\
\hline Cabernet & 177 & 107 & 91 & 65 & 94 & 13 \\
\hline Cabernet & 171 & 130 & 53 & 92 & 94 & 20 \\
\hline Cabernet & 211 & 89 & 79 & 115 & 84 & 16 \\
\hline Carbenet & 198 & 94 & 87 & 92 & 85 & 11 \\
\hline Chardonnay & 178 & 82 & 90 & 90 & 82 & 10 \\
\hline Chardonnay & 165 & 131 & 48 & 92 & 87 & 13 \\
\hline Chardonnay & 152 & 37 & 87 & 57 & 67 & 12 \\
\hline Chardonnay & 135 & 81 & 65 & 53 & 89 & 12 \\
\hline Chardonnay & 199 & 85 & 88 & 93 & 86 & 22 \\
\hline Chardonnay & 120 & 44 & 54 & 57 & 73 & 15 \\
\hline Chardonnay & 126 & 108 & 53 & 51 & 84 & 17 \\
\hline Chardonnay & 157 & 137 & 51 & 78 & 90 & 12 \\
\hline Chardonnay & 118 & 147 & 45 & 43 & 97 & 16 \\
\hline
\end{tabular}


GRÁFICO 1 - VALORES DA MÉDIA DO COLESTEROL TOTAL, POR GRUPO DE PACIENTES CONFORME AS UVAS DE CADA VINHO.

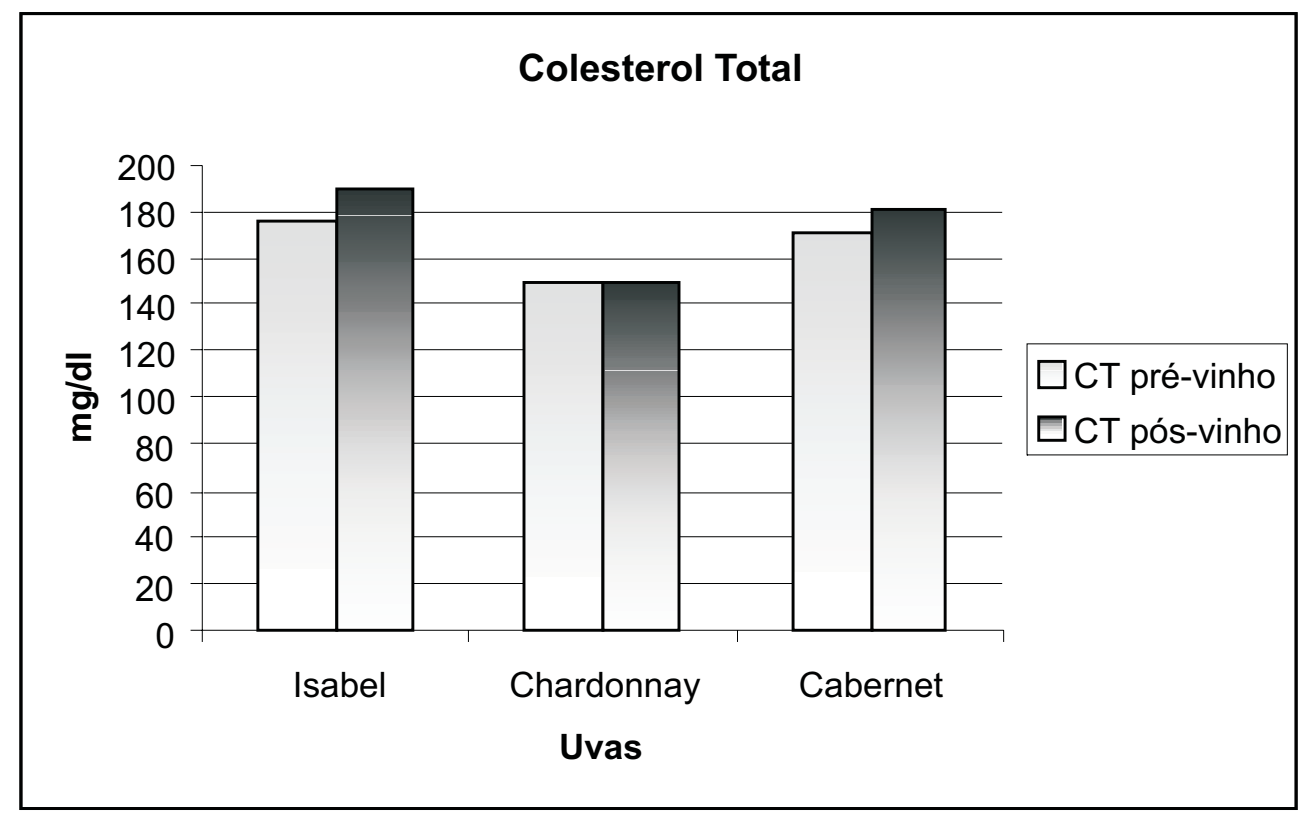

A taxa de triglicerídeos diminuiu $8 \%$ ao consumo de vinho de uvas Isabel, embora tenha aumentado em $4 \%$ ao grupo de vinho de uvas Chardonnay e 5\% em uvas Cabernet Sauvignon pós-vinho. (GRÁFICO 2).

GRÁFICO 2 - VALORES DA MÉDIA DOS TRIGLICERÍDEOS, POR GRUPO DE PACIENTES CONFORME AS UVAS DE CADA VINHO.

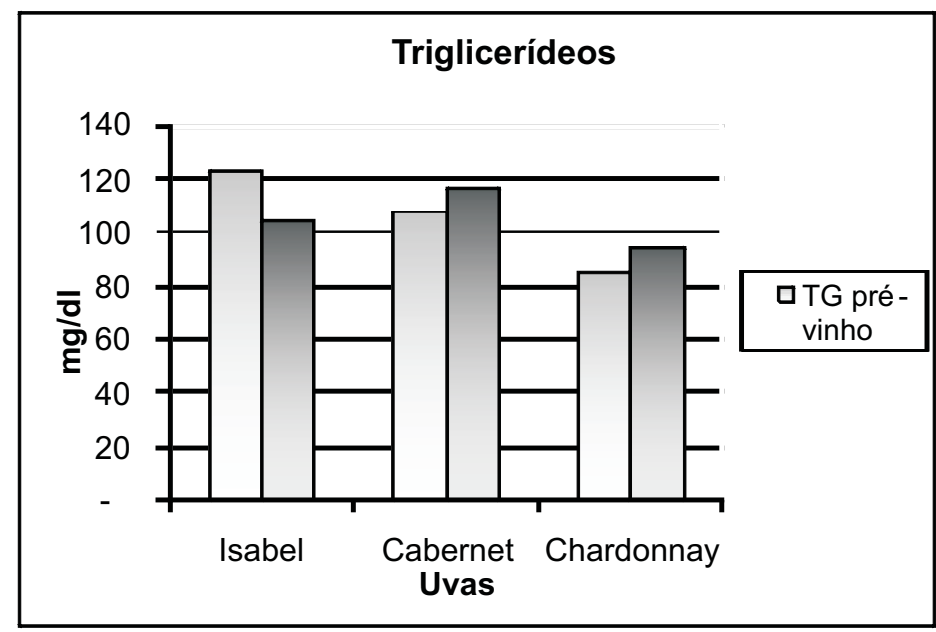

HDL - Colesterol apresentou melhora em ambos as uvas Isabel, Chardonnay e Carbenet Sauvignon após vinho (GRÁFICO 3), entre as dosagens plasmáticas, apenas consumidores do vinho de uva Isabel e Cabernet Sauvignon que a dosagem de HDLcolesterol aumentou de forma significativa. (GRÁFICO 4). 
GRÁFICO 3 - VALORES DA MÉDIA DO HDL - COLESTEROL, POR GRUPO DE PACIENTES CONFORME AS UVAS DE CADA VINHO.

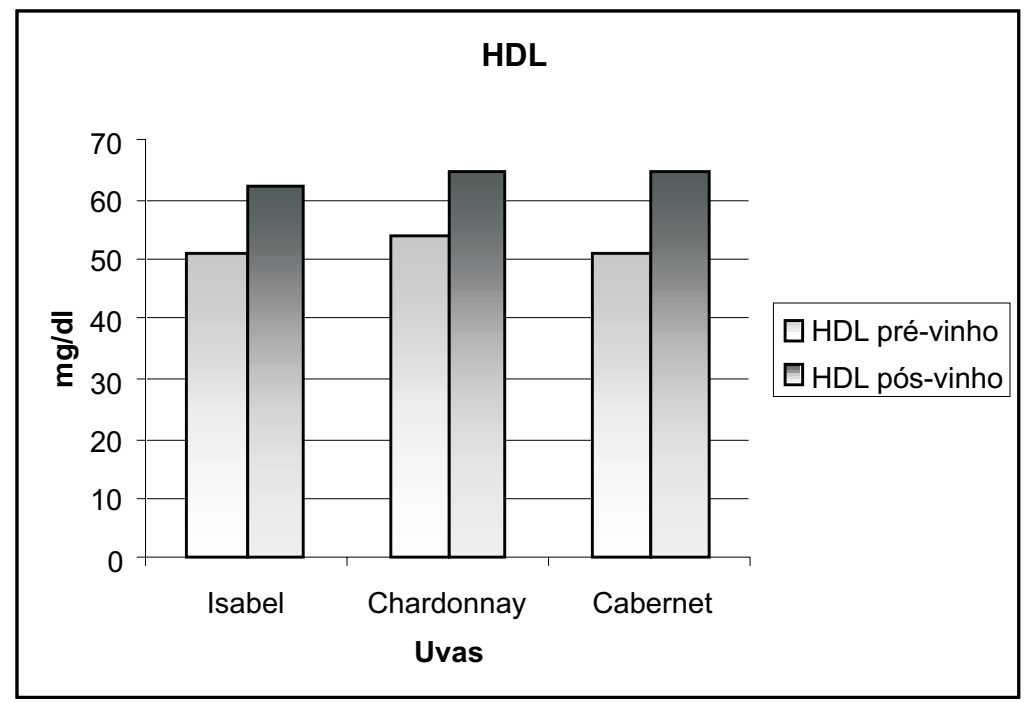

GRÁFICO 4 - VALORES SIGNIFICATIVOS (P<0,05 VS. PÓS-VINHO), DAS TAXAS DE HDL - COLESTEROL POR GRUPO DE PACIENTES CONFORME AS UVAS DE CADA VINHO.

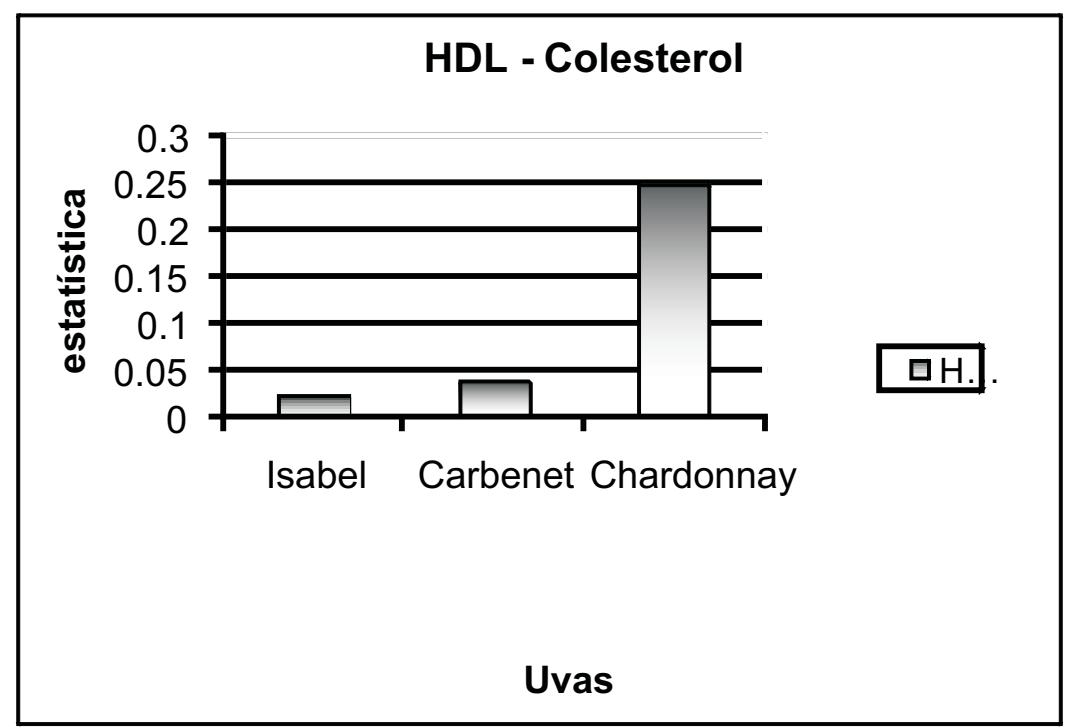

A média da taxa pós-vinho de LDL - Colesterol foi identificado que houve um aumento de $1,5 \%$ ao grupo que ingeriu vinho de uvas Isabel, entretanto, com uvas Chardonnay diminui 7\% e Cabernet Sauvignon diminui em 4\%. (GRÁFICO 5). 
GRÁFICO 5 - VALORES DA MÉDIA DO LDL - COLESTEROL, POR GRUPO DE PACIENTES CONFORME AS UVAS DE CADA VINHO.

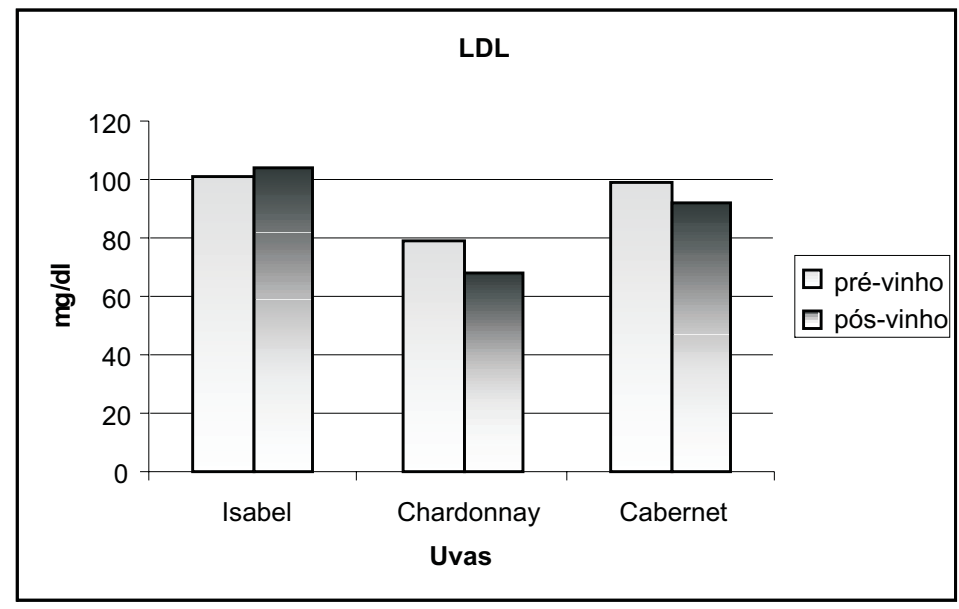

A glicose dos pacientes que ingeriram vinho de uva Chardonnay houve um declínio em 3\%, pós-vinho do grupo das uvas Isabel aumentou em 0,6\% e Cabernet Sauvignon aumentou em 2,3\%. (GRÁFICO 6)

GRÁFICO 6 - VALORES DA MÉDIA DA GLICOSE, POR GRUPO DE PACIENTES CONFORME AS UVAS DE CADA VINHO.

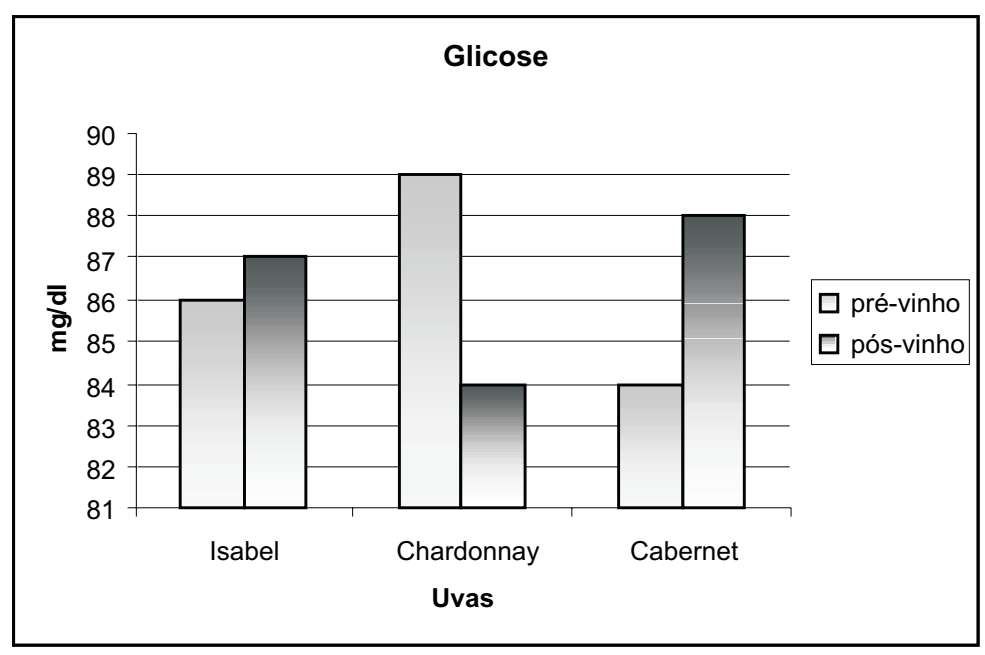

Não foi observada diferença significante entre as dosagens de colesterol total, triglicerídeos, LDL-Colesterol, glicemia e gama GT após vinho (gráfico 7). 
GRÁFICO 7 - VALORES DA MÉDIA DO GAMA GT, POR GRUPO DE PACIENTES CONFORME AS UVAS DE CADA VINHO.

Todos os valores das frações lipídicas foram analisados conforme os valores de referência de cada taxa lipídica (TABELA 3).

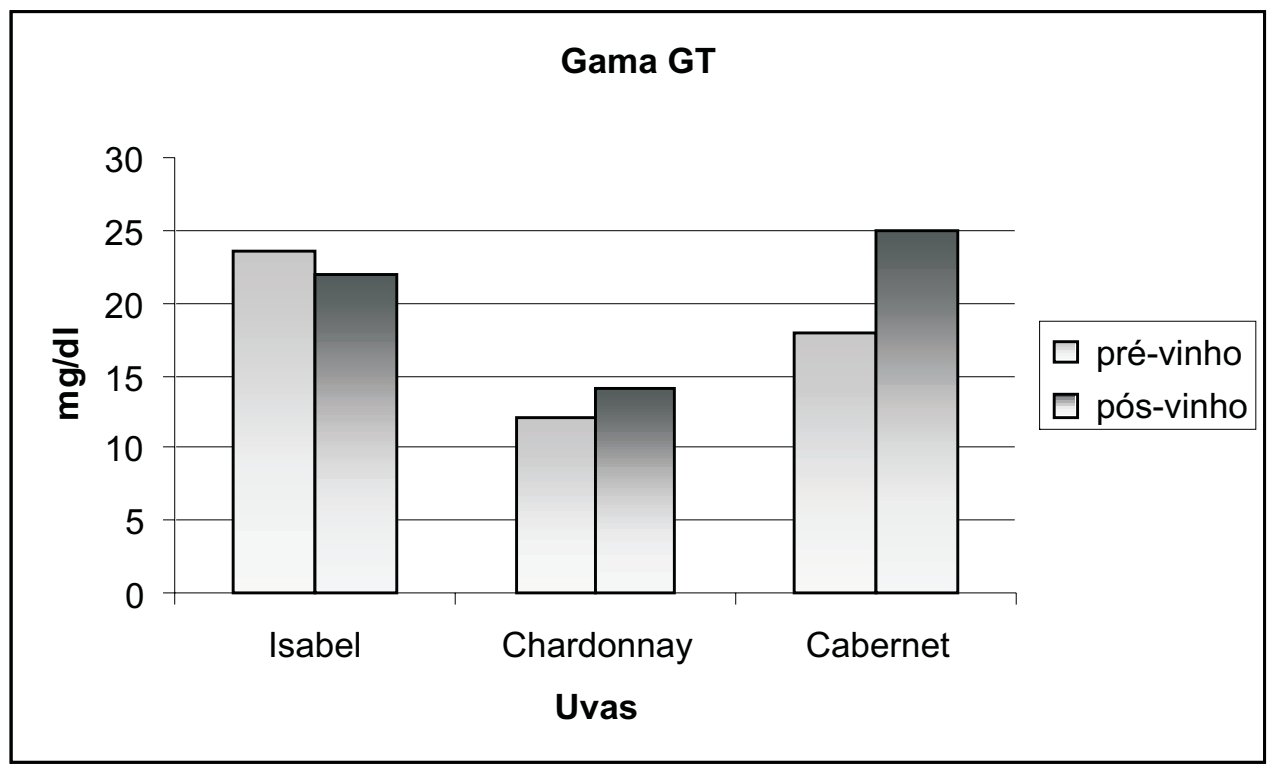

TABELA 3: VALORES DE REFERÊNCIAS DAS FRAÇÕES LIPÍDICAS.

\begin{tabular}{lll}
\hline Valores de referência dos lipídeos para indivíduos $>$ de 20 anos \\
\hline Lipídeos & Valores & Categoria \\
\hline Colesterol total & $<200 \mathrm{mg} / \mathrm{dL}$ & Ótimo \\
& $200-239 \mathrm{mg} / \mathrm{dL}$ & Limítrofe \\
& $>240 \mathrm{mg} / \mathrm{dL}$ & Alto \\
& $<100 \mathrm{mg} / \mathrm{dL}$ & Ótimo \\
& $100-129 \mathrm{mg} / \mathrm{dL}$ & Desejável \\
LDL $-\mathrm{C}$ & $130-159 \mathrm{mg} / \mathrm{dL}$ & Limítrofe \\
& $160-189 \mathrm{mg} / \mathrm{dL}$ & Alto \\
& $>190 \mathrm{mg} / \mathrm{dL}$ & Muito alto \\
& $<40 \mathrm{mg} / \mathrm{dL}$ & Baixo \\
& $>60 \mathrm{mg} / \mathrm{dL}$ & Alto \\
\hline HDL $-\mathrm{C}$ & $<150$ & Ótimo \\
& $150-200$ & Limítrofe \\
Triglicerídeos & $>500-499$ & Alto \\
& Muito alto
\end{tabular}

Fonte: III Diretrizes Brasileiras sobre Dislipidemias (2001) 


\section{DISCUSSÃO}

Os principais achados desse estudo foram: aumento de HDL - Colesterol, diminuição parcial de LDL - Colesterol e pequena elevação dos triglicerídeos.

$\mathrm{O}$ aumento de HDL significativo foi verificado no grupo que ingeriu vinho de uvas Isabel (vinho colonial) e vinho de uvas Cabernet Sauvignon, isso pode ser explicado pela quantidade de constituintes fenólicos que se encontra no vinho, principalmente nos tintos. Conforme estudos de Ishimoto et al., (2006) sugerem a existência de um sinergismo entre dois componentes, ou seja, o etanol e os constituintes fenólicos da uva, que atuariam em conjunto. Entretanto, segundo Blasi (2004), o etanol pode promover o efeito dos compostos fenólicos.

Magalhães Andrade (2006) também verificou em estudo de ingesta de vinho por 15 dias que HDL - Colesterol aumentou de forma significativa após vinho tinto. Vários estudos evidenciaram que o resveratrol pode elevar a concentração de HDL sanguíneo.

Por outro lado, sabe-se que o álcool aumenta os níveis de HDL-Colesterol, pois age no fígado e eleva a síntese de apolipoiproteína e aumenta a atividade da lipase lipoprotéica, que aumenta a formação dos níveis de HDL-colesterol.

O vinho tinto tem altos níveis de compostos fenólicos que favorecem múltiplos sistemas bioquímicos, assim como o aumento das lipoproteínas de alta densidade, atividade antioxidante, diminuição da agregação plaquetária, adesão endotelial, supressão do crescimento de células do câncer e promoção do oxido nítrico (De Loremier, 2000).

De acordo com os resultados obtidos, as taxas de LDL - Colesterol, diminuíram parcialmente com a ingesta dos vinhos varietais Cabernet Sauvignon e Chardonnay, isso decorrente aos flavonóides do vinho, principalmente o tinto que exerceram ação antioxidante, reduzindo a oxidação do LDL - Colesterol.

Golde et al., (1999) analisaram o papel do álcool com referência aos efeitos antioxidantes do vinho tinto contra a oxidação do colesterol LDL, o estudo in vitro confirmou o forte efeito antioxidante do vinho tinto, inibindo a oxidação do colesterol LDL, atrasando significativamente sua oxidação. Os pesquisadores concluíram que a diferença no potencial antioxidante entre vinho tinto e suco de uva tinto pode ser atribuída ao conteúdo e composição de polifenóis que difere entre as bebidas, pois a adição de álcool $12 \%$ ao suco de uva não teve efeito no atraso da oxidação do LDL.

Estudos de Giehl MR et al., (2007) demonstrou que a ingesta de vinho tinto após seis semanas, reduziu significativamente o colesterol LDL em $8 \%$, e houve um aumento importante e significativo no colesterol HDL de $17 \%$, relatam ainda que os benefícios do vinho tinto não são independentes do seu componente alcoólico.

As taxas de triglicerídeos aumentaram parcialmente no grupo que teve a ingesta de vinhos varietais Cabernet Sauvignon e Chardonnay, decorrente do teor alcoólico que influencia no aumento de frações dos triglicerídeos. O que torna o álcool desaconselhável em indivíduos com hipertrigliceridemia. No entanto, com o vinho colonial com uvas Isabel obteve um leve declínio nas taxas.

Assim confirmam-se efeitos protetores com consumo de vinho tinto mais do que vinho branco, pelas mudanças no perfil lipídico. $O$ efeito protetor do vinho tinto deve 
ser considerado, mas não devemos esquecer os seus outros efeitos como a embriaguez e as mudanças de comportamento, entre outros.

$\mathrm{Na}$ ausência de contra-indicação, o consumo diário de 200 a 300mL de vinho tinto (com $12 \%$ de graduação alcoólica) pode ser considerado um fator de proteção à saúde, principalmente a cardiovascular (ISHIMOTO; et al., 2006).

\section{CONCLUSÃO}

Foi possível indicar uma relação da ingestão de vinho e melhora de algumas das frações lipídicas.

Os níveis de HDL - Colesterol aumentou com a ingesta de vinho de uvas Cabernet Sauvignon, Chardonnay e Isabel. No qual apenas o vinho de a uva Isabel e Cabernet Sauvignon houve um aumento significativo $p<0,05$.

Ao realizar uma média por grupo de vinho, as análises do LDL - Colesterol demonstrou resultados adequados $(<100 \mathrm{mg} / \mathrm{dl})$, houve uma pequena diminuição nas frações lipídicas após a ingestão do vinho com uvas Cabernet Sauvignon e Chardonnay. Também diminuiu frações lipídicas de triglicerídeos após a ingesta de vinho com uvas Isabel. Apesar das diminuições das taxas, algumas medidas não foram significativas.

\section{REFERÊNCIAS}

ANDRADE, M. F.; PALMEIRA DE SOUZA, D. J.; et al.; Análise multivariada de parâmetros físico-químicos em amostras de vinhos tintos comercializados na região metropolitana do Recife. Quím. Nova v.31 n.2 São Paulo 2008

AZEVÊDO, L. C.; REIS, M. M.; SILVA, L. A.; ANDRADE, J. B.; Efeito da presença e concentração de compostos carbonílicos na qualidade de vinhos. Quím. Nova v.30 n.8 São Paulo, 2007.

BEHRENS,J.H.; SILVA, M.A.; Perfil sensorial de vinhos brancos varietais brasileiros através de análise descritiva quantitativa. Ciênc. Tecnol. Aliment. v.20 n.1 Campinas abr., 2000.

BERTAGNOLLO, S. M. M.; ROSSATO, S. B.; et al.; Influência da maceração carbônica e da irradiação ultravioleta nos níveis de trans-resveratrol em vinhos de uva cabernet sauvignon. Rev. Bras. Cienc. Farm. v.43 n.1 São Paulo jan./mar., 2007

BERTELLI, A. A. E; Wine, research and cardiovascular disease: Instructions for use. Department of Human Morphology, University of Milan, Milano, Italy. Atherosclerosis 195, p. 242-247, 2007.

BLASI. T. C.; Análise do consumo e constituintes químicos de vinho produzidos na quarta colônia de imigração italiana do Rio Grande do Sul e sua relação com as frações lipídicas sanguíneas. UFSM, 2004. 
Comitê da Sociedade Brasileira de Cardiologia. Consenso Brasileiro sobre dislipidemias: detecção, avaliação e tratamento. Arq Bras Cardiol, 1994.

DA LUZ, P. L.; Arquivos Brasileiros de Cardiologia Aterogênese. III Diretrizes Brasileiras sobre dislipidemias e Diretrizes de prevenção da aterosclerose do departamento de aterosclerose da sociedade brasileira de cardiologia. Rev. Sociedade Brasileira de Cardiologia, v.77, Suplemento III, p.10, 2001.

DAUDT, C. E.; SIMON, J. A.; Um método rápido para analise de glicose em mostos e sua qualificação em cultivares do Rio Grande do Sul. Cienc. Rural v.31 n.4 Santa Maria jul./ago., 2001.

DE LORIMIER, A. A.; Alcohol, wine and health. American Journal of Surgery. 180 (5):p. $357-61,2000$.

FERRARI, C. K. B.; Oxidative stress pathophysiology: Searching for an effective antioxidant protection. Intern. Med. J., v. 8, p. 175-184, 2001.

FORTES GRIS, E.; FALCÃO, I. D.; FERREIRA, E. A.; BORDIGNON, M. L.; Avaliação do tempo de meia-vida de antocianinas de uvas Cabernet Sauvignon em "Sorbet". B.CEPPA, Curitiba, v. 22, n. 2, jul./dez. 2004 B.CEPPA, Curitiba, v. 22, n. 2, p. 375-386, jul./dez., 2004.

GIEHL. M. R.; DAL BOSCO, S. M.; Eficácia dos flavonóides da uva, vinho tinto e suco de uva tinto na prevenção e no tratamento secundário da aterosclerose. Scientia Medica, Porto Alegre, v. 17, n. 3, p. 145-155, jul./set., 2007.

GOLDE PHM; SLOOTS L. M.; VERMEULEN W. P.; et al.; The role of alcohol in the anti low density lipoprotein oxidation activity of red wine. Atherosclerosis. 147:365-70, 1999.

GRONBAEK, M.; Wine, alcohol and cardiovascular risk: open issue. International Society on Thrombosis and Haemostasis 2: 2041-2., 2004.

ISHIMOTO, E. Y.; FERRARI, C. K. B.; TORRES, E. A. F. S.; Vinho: aspectos culturais, composição química e benefícios cardiovasculares. Nutrire: rev. Soc. Bras. Alim. Nutr.= J. Brazilian Soc. Food Nutr., São Paulo, SP, v. 31, n. 3, p. 127-141, dez., 2006.

JOHNSON, H.; The story of wine. London, UK: Octopus, 2004.

LINDBERG, M. L.; AMSTERDAM, E. A.; Alcohol, Wine, and Cardiovascular Health. Clin. Cardiol. 31, 8, 347-351., 2008.

LONA, A. A.; Vinhos - degustação, elaboração e serviço. 6aㅗ ed. Porto Alegre: AGE Editora, 2001. 
MAGALHÃES ANDRADE, A. C.; Ação do vinho tinto sobre o sistema nervoso simpático e função endotelial em pacientes hipertensos e hipercolesterolêmicos. São Paulo, 2006.

MALACRIDA, C. R.; MOTTA, S.; Antocianinas em suco de uva: composição e estabilidade. B.CEPPA, Curitiba, v. 24, n. 1, p. 59-82 jan./jun., 2006.

MANACH, C.; SCALBERT, A.; MORAND, C.; RÉMÉSY, C.; JIMÉNEZ, L.; Polyphenols: food sources and bioavailability. Am. J. Clin. Nutr., v. 79, n. 5, p. 727-747, 2004.

NASCIMENTO, A.C.; Vinho: saúde e longevidade. São Paulo, 2ª . ed. Idéia e Ação, 2005.

OLIVEIRA MAMEDE, M. E.; PASTORE, M.; Compostos fenólicos do vinho: estrutura e ação antioxidante. B.CEPPA, Curitiba, v. 22, n. 2, jul./dez. 2004 B.CEPPA, Curitiba, v. 22, n. 2, p. 233-252, jul./dez., 2004.

OPIE, L. H.; LECOUR, S.; The red wine hypothesis: from concepts to protective signalling molecules. The European Society of Cardiology. European Heart Journal 28, 16831693, 2007.

PARRET, B.; RUIDAVETS, J. B.; VIEU, C.; et al.; Alcohol consumption is associated with enrichment of high-density lipoprotein particles in polyunsaturated lipids and increased cholesterol etherification rate. Alcohol Clin. Exp. Res., 2002.

PENNA, N.G.; HECKTHEUER, L.H.; Vinho e Saúde: uma revisão. Infarma, v.16, no 12, 2004.

RIZZON, L.A.; MIELE, A.; Acidez na vinificação em tinto das uvas Isabel, carbenet sauvignon e carbenet franc. Cienc. Rural v.32 n.3 Santa Maria jun., 2002.

ROMA, M. G.; SANCHEZ POZZI, E. J.; Oxidative stress: A radical way to stop making bile. Annals of Hepatology 7(1): January-March: 16-33, 2008.

SAUTTER, C. K.; DENARDIN, S.; et al.; Determinação de resveratrol em sucos de uva no Brasil. Ciênc. Tecnol. Aliment. v.25 n.3 Campinas jul./set., 2005.

SCHWIMMER, J. B.; RONGHUI, X.; WINSTON, D.; Modest Wine Drinking and Decreased Prevalence of Suspected Nonalcoholic Fatty Liver Disease. HEPATOLOGY, Vol. 47, No. 6, 2008.

SOARES, M.; WELTER, L.; KUSKOSKI, E.M.; GONZAGA, L.; FETT, R.; Compostos fenolicos e atividade antioxidante da casca de uvas Niagara e Isabe. Rev. Brás. Frutic. v. 30 n. 1 Jaboticabal mar, 2008.

SOUSA NETO, J.A.; COSENZA, R.M.; Efeitos do Vinho no Sistema Cardiovascular. 
Revista Médica de Minas Gerais, vol. 4, No. 3, p.27-32, 1994.

SOUZA FILHO, M.J. Vinho e saúde: o estado da arte. MG, 2002.

UNIÃO DOS VITIVINICULTORES DO BRASIL. Consumo de vinhos no Brasil. Disponível em:<http://www.academiadovinho.com.br/produção.htm>. Acesso em 14 maio, 2009.

WATERHOUSE, A. L.; Wine phenolics. Ann New York academy of Sciences. 957:2136, University of California, USA, 2002. 